\title{
Article template makes your main claim in its brief title
}

\author{
Myles Axton ${ }^{1}$ \\ ${ }^{1}$ Genetics \& Genomics Next Editors
}

April 28, 2020

To use the actual template to write an Article, please use this link:

https://authorea.com/templates/article_template_for_genetics_genomics_next

Submissions should be made via the online manuscript tracking system. For technical help with the submission system, please contact ggn@wiley.com. Initial submission does not need to be formatted to Genetics E Genomics Next style For ease of evaluation and submission, the journal recommends an editable Word .docx or Authorea text document and a single merged PDF that includes all parts of main text and high-resolution figures embedded into the file. A suitable PDF will be constructed by uploading text and figures using the online manuscript tracking system. Genetics $\&$ Genomics Next does not impose word count and figure limits. Table 1 contains the journal's suggestions so that the manuscript is respectful of reader time and are readable by specialist and generalist alike.

\section{Acknowledgements}

Authors should list all funding sources here, please check Open Funder Registry. Contributions and material support from anyone not listed as an author should be acknowledged here, with permission from the contributor. Thanks to anonymous reviewers are not allowed.

\section{Conflict of Interest Statement}

All authors are required to declare if they have potential conflicts of interest related to the submission, or none. This declaration shall be published. Submitting authors shall confirm all co-authors agree with the final statement.

\begin{abstract}
What is known in the field, for a general readership. Define the area and knowledge for a specialist.

Explain the motivation and need for the research defined by the gap in existing knowledge.

State your main claim or finding. Support that with evidence, statistics and detail, mentioning essential methods and analytical techniques that provided the evidence.
\end{abstract}

State the meaning and significance of your new results for research in the field. 
End by suggesting realistic immediate implications and uses of your findings in your field and more broadly.

\section{Introduction}

Give credit to and cite all the primary research publications that lay the background to this work including those to be discussed in the Discussion. Give context as to whether these are essential methods and analytic strategies or experimental findings. Ensure that causation, correlation and conjecture

\section{Results}

Make the main claims in logical order, supported by display items and methods

\section{Discussion}

Summarize and evaluate the robustness and meaning of the main findings in light of existing publications. Be skeptical and discuss any limitations of the study and conditions where the results may or may not be applicable

\section{Materials and Methods}

\section{Methods and materials transparency}

Offer methods used in the analysis, and materials used to conduct the research to any researcher for purposes of reproducing the results or replicating the procedure. Indicate any restrictions on analytic methods including software, and tools and study materials available to other researchers. Specify how, where and when that material will be available. If an existing method or tool is used in the research, the authors are responsible for checking the license and state confirmation of permission.

To obtain Research Resource Identifiers (RRIDs): Use the Resource Identification Portal .

Design and analysis transparency

Authors are encouraged to review standards for disclosing key aspects of the research design and data analysis at http://www . equator-network. org/ and use those that are relevant for their research. Research reporting standards are widely adopted in our field, and exceeding their evolving requirements is essential to sustain the impact of genetics and genomics for research and for society. Here is the current list of reporting standards, vocabularies, models, schemas and databases that we recommend we recommend at FAIRsharing.org.

\section{Human studies and research participants}

Identify the ethics committee that approved the human study, and that the study conforms to recognized standards, for example: Declaration of Helsinki; US Federal Policy for the Protection of Human Subjects; or European Medicines Agency Guidelines for Good Clinical Practice. If no formal ethics committee is available, state that the research was carried out in accordance with recognized standards (e.g. the Declaration of Helsinki, as revised in 2013). 
Images and information from individual participants, including participants from patient registries and databases, will only be published where the authors have obtained the individual's free prior informed consent. Authors do not need to provide a copy of consent forms to the publisher but, in signing the author license to publish, authors are required to confirm that specific informed consent to publish the image has been obtained. Wiley has a standard patient consent form available for authors to use if required. This requirement to obtained informed consent applies whether or not patients are identifiable from the information presented in the submission.

\section{Animal studies}

For submissions involving animal studies, state the protocol and procedures employed were ethically reviewed and approved, and the name of the organization giving approval. State whether experiments were performed in accordance with relevant institutional and national guidelines and regulations for the care and use of laboratory animals:

US authors should cite compliance with the US National Research Council's Guide for the Care and Use of Laboratory Animals, the US Public Health Service's Policy on Humane Care and Use of Laboratory Animals, and Guide for the Care and Use of Laboratory Animals.

UK authors should conform to UK legislation under the Animals (Scientific Procedures) Act 1986 Amendment Regulations (SI 2012/3039).

EU authors should conform to Directive 2010/63/EU.

\section{Cell line authentication}

Declare where the cells were obtained, whether the cell lines have been tested and authenticated and the method by which the cells were tested. If cells were obtained directly from a cell bank that performs cell line characterizations and passaged in the user's laboratory for fewer than 6 months after receipt or resuscitation, re-authentication is not required.

\section{Data Availability Statement}

Please choose text from Table $\mathbf{3}$ and provide a citation to available data in the References list.

These sequence data have been submitted to the DDBJ/EMBL/GenBank databases under accession number XXXXX

Gene expression data (derived from microarrays or sequencing) has been deposited to a MIAME- or MINSEQEcompliant public repository like the Gene Expression Omnibus (GEO) with accession XXXXX

Protein Sequence Data should be submitted to UniProt with accession XXXXX

\section{References}

[terms in brackets will be removed before publication]

1. [article] Wood WG, Eckert GP, Igbavboa U, Muller WE. Statins and neuroprotection: a prescription to move the field forward. Ann N Y Acad Sci 2010; 1199:69-76.

2. [book] Hoppert, M. Microscopic techniques in biotechnology. Weinheim: Wiley-VCH; 2003. 
3. [dataset]Authors; Year; Dataset title; Data repository or archive; Version (if any); Persistent identifier (e.g. DOI)

4. [URI, GWAS summary statistics] Savage, J.E. et al. Genome-wide association meta-analysis in 269,867 individuals identifies new genetic and functional links to intelligence https://www.ebi.ac.uk/gwas/studies/GCST006250 (2018)

5. [supplementary data] Jagadeesan, A. et al. MDS/PCA plots within West Africa https://doi.org/10.6084/m9.figshare.5640931 (2017)

\section{Tables (each table complete with title and footnotes)}

\begin{tabular}{|c|c|c|c|c|c|}
\hline Number of words, references or display items & $\begin{array}{l}\text { Arti- } \\
\text { cle }\end{array}$ & $\begin{array}{c}\text { Per- } \\
\text { spec- } \\
\text { tive }\end{array}$ & $\begin{array}{c}\text { Anal- } \\
\text { ysis }\end{array}$ & $\begin{array}{c}\text { Re- } \\
\text { source }\end{array}$ & $\begin{array}{l}\text { Technical } \\
\text { Report }\end{array}$ \\
\hline Abstract & 200 & 200 & 200 & 200 & 200 \\
\hline $\begin{array}{l}\text { Introduction }+ \text { Results }+ \text { Discussion for original } \\
\text { research; main text for others }\end{array}$ & ¡4000 & ¡3000 & $¡ 2000$ & ¡4000 & ¡4000 \\
\hline Methods & $¡ 4000$ & none & $¡ 4000$ & ¡ 4000 & ¡4000 \\
\hline each Figure Legend & 500 & 300 & 300 & 500 & 500 \\
\hline References & ¡ 100 & ¡ 100 & ¡ 100 & i 100 & ¡ 100 \\
\hline Figures + Tables & i 8 & i 4 & i 4 & i 8 & i 8 \\
\hline
\end{tabular}

Table 1: Recommended article types and sizes. We have no formal limits, but please be concise since referee and reader time is the most valuable part of the research economy.

\section{Figures}

\section{Appendices}

Genetics $\mathscr{E}$ Genomics Next encourages authors to mark up supporting datasets and supporting information with rich metadata and deposit them with a CC BY open attribution license in an appropriate repository such as Authorea, CoS.io, Dryad, Figshare or zenodo and to cite the whole collection once (and individual datasets as needed) from the References list.

Unformatted supporting information is less useful to readers, but it does provide a degree of provenance and context to the article. It can be hosted online where it appears without editing or typesetting as supplementary tables and figures, videos or datasets. Please see Wiley's FAQs on supporting information. 
Sort by: alphabetical order publication year frequency of citation (within this paper) number (original)
Turn citation typing off

1. United Nations Human Settlements Programme (2003) The challenge of slums: Global report on human settlements 2003. London: Earthscan Publications Ltd. Link (CiTO: obtains background from, Report, Book, Online Document, not peer reviewed)

2. Riley LW, Ko AI, Unger A, Reis MG (2007) Slum health: Diseases of neglected populations. BMC Int Health Hum Rights 7: 2. DOI PubMed PubMedCentral (CiTO: obtains background from, shares authors with, Opinion, Journal Article, peer reviewed)

3. Sclar ED, Garau P, Carolini G (2005) The 21st century health challenge of slums and cities. Lancet 365: 901-903. DOI PubMed (CiTO: obtains background from, Opinion, Journal Article, peer reviewed)

Figure 1: Semantic identification of types of citation. Image credit: CC-BY Shotton, D. et al. 2009 https://doi.org/10.1371/journal.pcbi.1000361 Figure number should be followed by a one sentence main figure heading. The subpanel description and the rest of the legends should be concise but comprehensive - the figure and its legend must be understandable without reference to the text. Include definitions of any symbols used and define/explain all abbreviations and units of measurement. Statistical tests, assumptions, parameters and statistics should be exactly stated. Scale bars should be explained, with units. 


\author{
CRediT \\ Conceptualiza- \\ tion
}

Data curation

experimental contribution

Formal analysis

intellectual contribution

$\begin{array}{cc}\text { Funding } & \text { organizational contribution } \\ \text { acquisition } & \text { experimental contribution }\end{array}$

Methodology

experimental contribution

Project organizational contribution administration
Resources
experimental contribution

Software

Supervision

Validation

experimental contribution https://sparontologies.github.io/scoro/cur

experimental contribution

intellectual contribution

Visualization

Writing original draft

Writing review \& editing
SCORO named individuals conceives project

designs experiments

formulates research questions provides advice

https://sparontologies.github.io/scoro/current/scoro.html\# maintains IT infrastructure

provides existing data

provides software

provides technical support analyses data interprets results undertakes modelling https://sparontologies.github.io/scoro/current/scoro.html\#

collects data performs experiments processs data

builds and/or maintains instruments creates novel reagents develops methodology https://sparontologies.github.io/scoro/current/scoro.html\#

https://sparontologies.github.io/scoro/current/scoro.html\# https://sparontologies.github.io/scoro/current/scoro.html\#

obtains and/or prepared specimens

provides reagents, specimens or materials

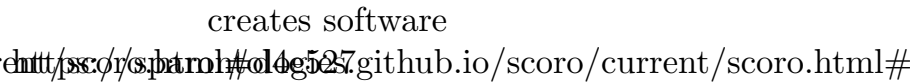
https://sparontologies.github.io/scoro/current/scoro.html\# performs experiments provides existing data processs data analyses data provides advice undertakes modelling prepares illustrations prepares supplementary information

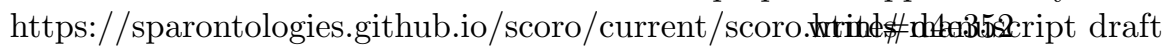

prepares supplementary information

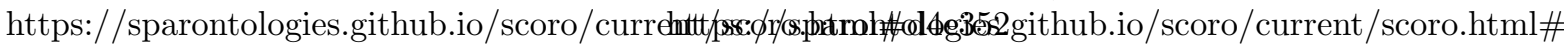
https://sparontologies.github.io/scoro/current/scoro.html\#

Table 2: Author contributions and equality statements All authors should have contributed to the manuscript substantially and have agreed to the final submitted version. The submitting author must provide an ORCiD when submitting a manuscript. We would be delighted if all authors provide an ORCiD upon acceptance for publication. This takes around 2 minutes per person to complete. Find more information here. Each author can claim one or more of the fourteen Article Author Contributions on the left, that are explained here in terms of the SCORO ontology. In the case of joint authorship, a numbered footnote should be added to the author listing, e.g. 'X. Liu, Y. Sanchez and Z. Schwartz made equal contributions' or 'O. Mkwanza and P. Ollo jointly supervised the work.' 
Availability of data

Data openly available in a public repository that issues datasets with DOIs

Data openly available in a public repository that does not issue DOIs

Data derived from public domain resources

Embargo on data due to commercial restrictions

Data available on request due to privacy/ethical restrictions
Template for data availability statement

The data that support the findings of this study are openly available in [repository name e.g "figshare"] at http://doi.org/[doi], reference number [reference number].

The data that support the findings of this study are openly available in [repository name] at [URL], reference number [reference number].

The data that support the findings of this study are available in [repository name] at [URL/DOI], reference number [reference number]. These data were derived from the following resources available in the public domain: [list resources and URLs]

The data that support the findings will be available in [repository name] at [URL / DOI link] following an embargo from the date of publication to allow for commercialization of research findings.

The data that support the findings of this study are available on request from the corresponding author. The data are not publicly available due to privacy or ethical restrictions.

Table 3: Data availability statement options. Table legends: should be self-contained and complement, but not duplicate, information contained in the text. They should be supplied as editable files, not pasted as images. Legends should be concise but comprehensive - the table, legend, and footnotes must be understandable without reference to the text. All abbreviations must be defined in footnotes. Footnote symbols:,,$+++ \S$, I, should be used (in that order) and ${ }^{*},{ }^{* *},{ }^{* * *}$ should be reserved for P-values. Statistical tests with essential parameters, and measures such as SD or SEM should be identified in the headings. 